\title{
ALEXANDER DUALITY AND HUREWICZ FIBRATIONS
}

\author{
STEVEN C. FERRY
}

\begin{abstract}
We explore conditions under which the restriction of the projection map $p: S^{n} \times B \rightarrow B$ to an open subset $U \subset S^{n} \times B$ is a Hurewicz fibration. As a consequence, we exhibit Hurewicz fibrations $p: E \rightarrow I$ such that: (i) $p: E \rightarrow I$ is not a locally trivial bundle, (ii) $p^{-1}(t)$ is an open $n$-manifold for each $t$, and (iii) $p \circ$ proj: $E \times R^{1} \rightarrow I$ is a locally trivial bundle. The fibers in our examples are distinguished by having nonisomorphic fundamental groups at infinity. We also show that when the fibers of a Hurewicz fibration with open $n$-manifold fibers have finitely generated $(n-1)$ st homology, then all fibers have the same finite number of ends. This last shows that the punctured torus and the thrice punctured two-sphere cannot both be fibers of a Hurewicz fibration $p: E \rightarrow I$ with open 2-manifold fibers.
\end{abstract}

\section{INTRODUCTION}

All spaces considered in this paper will be locally compact metric spaces. By a fibration we will mean a map $p: E \rightarrow B$ which is a Hurewicz fibration. By a bundle, we will mean a map $p: E \rightarrow B$ which has a local product structure, i.e., such that for every $b \in B$ there exist a neighborhood $U$ of $b$, a space $F$, and a homeomorphism $h: p^{-1}(U) \rightarrow F \times U$ such that proj $h=p$, where proj: $F \times U \rightarrow U$ is the projection. A bundle or fibration $p$ has $n$-manifold fibers if $p^{-1}(b)$ is an $n$-dimensional manifold for each $b \in B$. Throughout this paper, we will assume that the base space $B$ is a finite polyhedron.

We wish to study the relation between fibrations and bundles with $n$-manifold fibers. A number of cases are known in which the two notions coincide. This was proved by Raymond in [R] for the case in which the fibers are closed 2manifolds and by T. A. Chapman and the author in case the fibers are closed $n$-manifolds with $n>4$ [ChF]. In dimension 4 , the same result is implied by work of $F$. Quinn $\left[Q_{2}\right]$ (see Proposition 1.4). In dimension 3, the outcome depends on the classical Poincare conjecture. This is explained in Proposition 1.5 .

Much of the motivation for this study was supplied by the close relationship between fibrations with polyhedral fibers and automorphism groups of PL and topological manifolds $[\mathrm{H}, \mathrm{W}]$. The theorem from $[\mathrm{ChF}]$ quoted above has a PL

Received by the editors June 10, 1989 and, in revised form, August 11, 1989.

1980 Mathematics Subject Classification (1985 Revision). Primary 57N15, 55R05, 55R10.

Key words and phrases. Hurewicz fibration, Čech cohomology, acyclic space.

The author was partially supported by NSF Grants DMS-8502777 and DMS-8702991. 
analog $\left[\mathrm{Ch}_{2}\right]$ which globalizes the topological invariance of Whitehead torsion. Such theorems can be thought of as rigidity theorems-that a topological homeomorphism preserves stable PL structure or that a (topological) fibration has a well-defined PL spine.

For noncompact manifolds, Chapman $\left[\mathrm{Ch}_{1}\right]$ proved that the two notions nearly coincide for proper fibrations. (A map $p: E \rightarrow B$ is called a proper fibration if for each $b \in B$ there exist a neighborhood $U$ of $b$, a space $X$, and proper maps $r: X \times U \rightarrow p^{-1}(U)$ and $i: p^{-1}(U) \rightarrow X \times U$ for which $r \circ i=$ id and $r(X \times\{b\})=p^{-1}(b)$ for all $b \in U$. A similar condition has been studied by Dold [D]. Note that a proper fibration is not required to be a proper map.) Chapman showed that a proper fibration with open (i.e., connected, noncompact, and without boundary) $n$-manifold fibers is a bundle whenever $n>4$ and the fibers are simply-connected at infinity. He proved a similar theorem when the fibers are manifolds with boundary and the restriction to the boundary is a bundle.

In the same paper, Chapman showed that the hypothesis at infinity can be dropped if one is willing to cross the total space of the fibration with $D^{2}$. Chapman and West, in unpublished work, have shown that the hypothesis at infinity is entirely superfluous for high-dimensional fibers. It seems natural to ask whether Chapman's "proper fibration" hypothesis can be weakened. In particular, one can ask whether every Hurewicz fibration with $n$-manifold fibers is a bundle.

In this generality, counterexamples abound. Let $D^{n}$ be the $n$-ball and let proj: $D^{n} \times I \rightarrow I$ be projection. If $A$ is any subset of $\partial D^{n} \times I$, then $\operatorname{proj} \mid\left(D^{n} \times I-A\right):\left(D^{n} \times I-A\right) \rightarrow I$ is a Hurewicz fibration (see Examples 1.6 for details). We will refer to a Hurewicz fibration $p: E \rightarrow B$ with connected open $n$-manifold fibers as an open $n$-manifold fibration. If $p: E \rightarrow I$ is an open $n$-manifold fibration with $M=p^{-1}(0)$ and $N=p^{-1}(1)$, we will say that $M$ deforms to $N$ through open $n$-manifolds. Examples 1.6 suggest searching for interesting deformations by studying sets $A \subset S^{n} \times I$ such that proj|: $\left(S^{n} \times I-A\right) \rightarrow I$ is a fibration. This case is more delicate than the one above. Since the various fibers $(\operatorname{proj} \mid)^{-1}(t)$ are homotopy equivalent, Alexander duality dictates that the spaces $A_{t}=A \cap S^{n} \times\{t\}$ must have isomorphic Čech cohomology groups.

The notion of a homology fibration was defined by D. McDuff in [Mc] and then redefined in a stronger way by McDuff and Segal in [McS]. We restate the definition from $[\mathrm{McS}]$ using Čech cohomology to allow for the possibility of non-ANR fibers. Recall that $f: X \rightarrow Y$ is proper if $f^{-1}(K)$ is compact for each compact subset $K$ of $Y$.

Definition. A proper map $p: X \rightarrow B$ from a finite-dimensional metric space to a finite polyhedron is a Čech cohomology fibration if each $b \in B$ has arbitrarily small contractible closed neighborhoods $N$ such that the inclusion $p^{-1}\left(b^{\prime}\right) \rightarrow$ $p^{-1}(N)$ is a Čech cohomology equivalence for each $b^{\prime} \in N$. 
The next definition gives the standard tameness criterion for embeddings of compacta into manifolds.

Definition. A compact subset $X$ of a compact manifold $M$ is said to be 1-LCC (1-locally co-connected) if for each $\varepsilon>0$ there is a $\delta>0$ so that every map $f: S^{1} \rightarrow M-X$ with $\operatorname{diam}\left(f\left(S^{1}\right)\right)<\delta$ extends to a map $\bar{f}: D^{2} \rightarrow M-X$ with $\operatorname{diam}\left(\bar{f}\left(D^{2}\right)\right)<\varepsilon$. An embedding $i: X \rightarrow M$ is 1-LCC if $i(X)$ is 1-LCC in $M$.

Here is our main theorem:

Theorem 1. Let $p: X \rightarrow B$ be a proper map from a finite-dimensional metric space to a finite polyhedron. Then the following conditions are equivalent:

(i) $p$ is a Čech cohomology fibration.

(ii) There is a fiberwise codimension-three fiber-preserving ( $\equiv f . p$.) fiberwise 1-LCC embedding $i: X \rightarrow S^{n} \times B$ for some $n$ such that $\operatorname{proj}_{B} \mid\left(S^{n} \times B-i(X)\right)$ is a Hurewicz fibration.

Example 1. Let $K$ be a 2-complex with $\pi_{1}(K) \neq 1$ and $H_{*}(K)=H_{*}(\mathrm{pt})$. Let the cone on $K$ be given as $C K=K \times I / K \times\{1\}$, where $I=[0,1]$. Let $p: C K \rightarrow I$ be projection onto the $I$-coordinate. According to Theorem 1 there is a fiberwise 1-LCC fiberwise codimension-three embedding $i: C K \rightarrow S^{n} \times I$ so that $\operatorname{proj}_{I} \mid\left(S^{n} \times I-C K\right)$ is a Hurewicz fibration. This fibration has open $n$-manifold fibers but cannot be a bundle, since one fiber is $R^{n}$, while the others are not simply-connected at infinity.

Corollary 1. There are Hurewicz fibrations with open n-manifold fibers which are not locally trivial bundles. In fact, different fibers in such a fibration can have different fundamental groups at infinity. Such fibers are not even proper homotopy equivalent.

Notation. If $i: X \rightarrow S^{n} \times B$ is an embedding, we will use $i_{l}: X \rightarrow S^{n+l} \times B$ to denote the embedding induced by the standard inclusion $S^{n} \subset S^{n+l}$.

We will prove that (i) $\Rightarrow$ (ii) in Theorem 1 by showing the following:

Theorem 2. Let $p: X \rightarrow B$ be a Čech cohomology fibration and let $i: X \rightarrow S^{n} \times$ $B$ be an f.p. fiberwise 1-LCC fiberwise codimension-three embedding, $n \geq 5$. If $k=\operatorname{dim} B$, then for $l \geq n+4 k+4$

(i) $\operatorname{proj}_{B} \mid:\left(S^{n+l} \times B-i_{l}(X)\right) \rightarrow B$ is a Hurewicz fibration and

(ii) the composition $\left(S^{n+l} \times B-i_{l}(X)\right) \times R^{2} \rightarrow\left(S^{n \times l} \times B-i_{l}(X)\right) \rightarrow B$ is a locally trivial bundle.

Since a fiber-preserving retract of a locally trivial bundle is a Hurewicz fibration, (ii) implies (i) in this theorem.

Corollary 2. There are Hurewicz fibrations with open manifold fibers which are not locally trivial bundles but which become locally trivial bundles upon stabilization by crossing with a euclidean space. 
Our proof of the (easy) implication that (ii) $\Rightarrow$ (i) in Theorem 1 rests on the following duality theorem which mixes Alexander and Poincaré-Lefschetz dualities.

Theorem 3. Let $M^{n}$ be a compact orientable PL manifold with boundary and let $X \subset M^{n}$ be compact. Write $\partial M=P \cup Q$, where $P$ and $Q$ are codimension- 0 submanifolds of $\partial M$ and $\partial P=\partial Q$. Then

$$
\check{H}^{k}(M, Q \cup X) \cong H_{n-k}(M-X, P-P \cap \partial X) \text { for all } k \geq 0 .
$$

Here, we write $\partial X=X \cap \partial M$.

Our final result shows that even though proper homotopy type is not preserved by deformation of open $n$-manifolds, some properties at $\infty$ are invariant.

Theorem 4. (i) Let $M$ be a compact n-manifold with boundary. If $M$ deforms to another compact $n$-manifold with boundary $N$ through compact $n$-manifolds with boundary, then there is a 1-1 correspondence between boundary components of $M$ and boundary components of $N$. Moreover, corresponding boundary components are homology equivalent.

(ii) Let $M$ be a connected open n-manifold with $\operatorname{dim}_{\mathbb{F}} H_{n-1}(M ; \mathbb{F})<\infty$, where $\mathbb{F}$ is a field such that $M$ is orientable over $\mathbb{F}$. If $M$ deforms to an open $n$-manifold $N$ through open $n$-manifolds, then $M$ and $N$ have the same number of ends.

Example 2. Let $W^{4}$ be a contractible 4-manifold with nonsimply connected homology sphere boundary. Let $N$ be a regular neighborhood of a point $x \in$ $\partial W$ and let $\partial N=\partial N_{+} \cup \partial N_{-}$, where $\partial N_{+}=\partial N \cap \partial W$. Let $c$ be the map $c: W \rightarrow W / \operatorname{cl}(W-N)=N / \partial N_{-} \cong D^{4}$. Let $M(c)$ be the mapping cylinder of $c$ and let $p: M(c) \rightarrow[0,1]$ be the natural projection. Since $c$ is cell-like, $p$ is a Hurewicz fibration. See [H] or apply the proof of Proposition 1.5 to the space obtained by doubling $M(c)$ along the boundary in each fiber and then recall that a fiber-preserving retract of a Hurewicz fibration is a Hurewicz fibration. It is also worth noting that $p \circ$ proj: $M(c) \times I \rightarrow[0,1]$ is a bundle with fiber $D^{5}$.

Corollary 3. The 2-torus with one puncture and the 2-sphere with three punctures do not deform to each other, even though these manifolds become homeomorphic when multiplied by $R^{1}$.

Question. When do open $n$-manifolds $M$ and $N$ deform to each other through open $n$-manifolds? When do compact $n$-manifolds with boundary $M$ and $N$ deform to each other through compact $n$-manifolds with boundary?

I would like to thank Professor A. Dold for asking (during a hike at Oberwolfach) whether an open manifold fibration is always a locally trivial fiber bundle. I would also like to thank T. Moore for helpful suggestions. 


\section{SOME ELEMENTARY CONSTRUCTIONS}

In this section, we prove some of the results described in the introduction. Many of these results are known to experts, but the exposition may help the reader to become oriented to what follows.

The basic tool for proving that a Hurewicz fibration is a bundle is a theorem of Dyer and Hamstrom which is itself a corollary to a selection theorem of $\mathrm{E}$. Michael.

Definition [DH, p. 104]. A mapping $f$ of a metric space $X$ onto a metric space $Y$ is said to be completely regular provided that for each point $y$ of $Y$ and positive number $\varepsilon$ there is a positive number $\delta$ such that if $x$ is a point of $Y$ and $d(x, y)<\delta$, then there is a homeomorphism of $f^{-1}(x)$ onto $f^{-1}(y)$ which moves no point as much as $\varepsilon$.

Theorem 1.1 (Dyer-Hamstrom [DH, Note, p. 109]). If $Y$ is finite-dimensional, $X$ is compact, and $f: X \rightarrow Y$ is completely regular with the homeomorphism group of $f^{-1}(y)$ locally contractible for each $y \in Y$, then $f: X \rightarrow Y$ is a locally trivial fiber bundle.

This meshes nicely with the following theorem of Edwards and Kirby.

Theorem 1.2 [EK, Corollary 1.1]. If $M$ is a compact topological manifold of any dimension, then the homeomorphism group of $M$ is locally contractible.

Thus, to show that a fibration with finite-dimensional base and closed $n$ manifold fibers is a locally trivial bundle, one need only show that nearby pointinverses are homeomorphic via small homeomorphisms.

Proposition 1.3 [ChF]. If $n \geq 5$ is fixed, $B$ is a locally finite-dimensional locally path-connected space, and $p: E \rightarrow B$ is a Hurewicz fibration with $p^{-1}(b) a$ closed $n$-manifold for each $b \in B$, then $p$ is the projection map of a locally trivial fiber bundle.

Proof. We sketch the proof. The reader is referred to [ChF] for details. Nearby fibers in a Hurewicz fibration with compact total space are homotopy equivalent via homotopy equivalences which are close to the identity in the topology of the total space [Du, p. 400]. The main theorem of [ChF] shows that small homotopy equivalences between high-dimensional manifolds are approximable by homeomorphisms. Combining this with the theorems of Edwards-Kirby and Dyer-Hamstrom proves Proposition 1.3.

Proposition 1.4. If $B$ is a locally finite-dimensional, locally path-connected space, and $p: E \rightarrow B$ is a Hurewicz fibration with $p^{-1}(b)$ a closed 4-manifold for each $b \in B$, then $p$ is the projection map of a locally trivial fiber bundle.

Proof. This is the 4-manifold version of Proposition 1.3. We state it separately because the proof is somewhat different.

If $b$ and $b^{\prime}$ are nearby points of $B$, let $\alpha: I \rightarrow B$ be an arc with $\alpha(0)=b$ 
and $\alpha(1)=b^{\prime}$. Let $\alpha^{*} E$ be the pullback of $p$ over $\alpha$ :

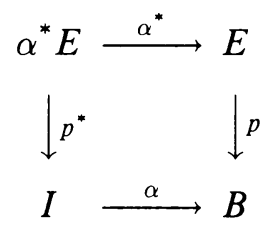

$\alpha^{*} E$ is an ANR homology manifold, since $\alpha^{*} E \times S^{1}$ satisfies the conditions of Proposition 1.3 with 5-manifold fibers and is therefore a locally trivial bundle. Using lifting functions as in [Du, p. 400], one sees that $\alpha^{*} E$ is a thin $h$-cobordism (in the sense of $\left[\mathrm{Q}_{1}\right]$ ) of ANR homology manifolds. Since $\alpha^{*} E$ has nonempty manifold boundary, the obstruction to resolution $\left[\mathrm{Q}_{2}\right]$ vanishes and $\alpha^{*} E$ can be resolved relative to its ends to obtain a thin $h$-cobordism joining $\left(p^{*}\right)^{-1}(0)$ to $\left(p^{*}\right)^{-1}(1)$. An application of the thin $h$-cobordism theorem produces a homeomorphism $h:\left(p^{*}\right)^{-1}(0) \rightarrow\left(p^{*}\right)^{-1}(1)$ which approximates the original homotopy equivalence from $p^{-1}(b)$ to $p^{-1}\left(b^{\prime}\right)$. This demonstrates the complete regularity of $p$ and completes the proof.

Proposition 1.5. If $\Sigma^{3}$ is a homotopy 3-sphere, then there is a Hurewicz fibration $p: E \rightarrow I$ with closed 3-manifold fibers so that $p^{-1}(0)=\Sigma^{3}$ and $p^{-1}(1)=S^{3}$. Proof. Let $D^{3} \subset \Sigma^{3}$ be a small standard disk and let $c: \Sigma^{3} \rightarrow S^{3}$ be the map which crushes closure $\left(\Sigma^{3}-D^{3}\right)$ to a point. Let $E$ be the mapping cylinder of $c, E=\Sigma^{3} \times I \amalg S^{3} / \sim$, where $c(x, 1) \sim c(x)$. Projection onto the $I$-factor induces a map $p: E \rightarrow I$ with $p^{-1}(0)=\Sigma^{3}$ and $p^{-1}(1)=S^{3}$. It remains to show that this map is a Hurewicz fibration (see Figure 1).

Our plan is to cross $E$ with $S^{2}$ to form a map with 5-manifold fibers. We will show that $p \circ$ proj: $E \times S^{2} \rightarrow I$ is completely regular, which will show that it is a locally trivial bundle map. It then follows that $p: E \rightarrow I$ is a Hurewicz fibration, since one easily checks that a fiber-preserving retract of a Hurewicz fibration is a Hurewicz fibration.

That $p \circ$ proj: $E \times S^{2} \rightarrow I$ is completely regular is checked using Siebenmann's CE approximation theorem [S]. This theorem says that every cell-like map between closed manifolds of dimension $\geq 5$ is uniformly approximable by homeomorphisms. We need only check complete regularity near $p^{-1}(1)$. Since the point-inverses of $c$ are contractible, $c$ is cell-like. Crossing with $S^{2}$ satisfies the dimension condition, so the cell-like map $c \times$ id: $\Sigma^{3} \times S^{2} \rightarrow S^{3} \times S^{2}$ is uniformly approximable by homeomorphisms. $E \times S^{2}$ is the mapping cylinder of $c \times$ id, and the projection $\Sigma^{3} \times S^{2} \times\{t\} \rightarrow S^{3} \times S^{2}$ is small in the topology of the total space for $t$ near 1 . This shows that $p \circ$ proj: $E \times S^{2} \rightarrow I$ is completely regular and proves Proposition 1.5.

In contrast to the above, it is easy to produce examples of Hurewicz fibrations which are not bundles when the fibers are manifolds with boundary.

Examples 1.6 (see Figure 2). (i) F. Raymond pointed out in [R] that the pro- 


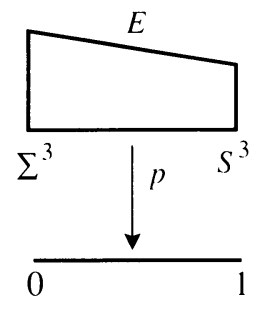

FIGURE 1
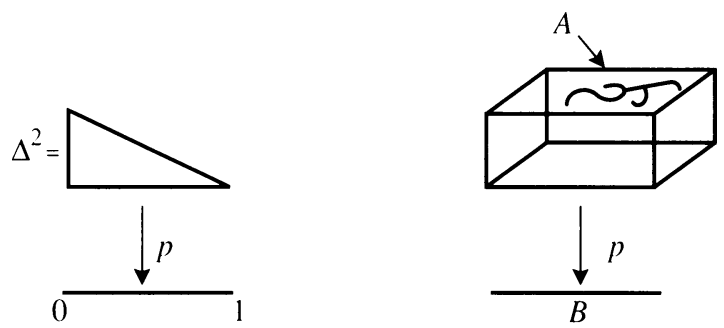

FIGURE 2

jection from a 2-simplex to a 1-simplex is a Hurewicz fibration but is not a bundle.

(ii) If $p: D^{n} \times B \rightarrow B$ is the projection, the restriction $p \mid:\left(D^{n} \times B-A\right) \rightarrow B$ is a Hurewicz fibration for any $A \subset \partial D^{n} \times B$. If $g: Z \times I \rightarrow B$ is a map and $G: Z \times I \rightarrow D^{n} \times B$ is a lifting, then $(1-t) \cdot G$ is a lifting into $D^{n} \times B-A$, where - denotes scalar multiplication in the $D^{n}$-factor. In words, if the lifting ducks inside quickly, it misses any disturbance on the boundary.

\section{THE PROOF OF THEOREM 2}

We begin by proving Theorem 2 . Since every finite-dimensional metric space has a 1 -LCC embedding in some $S^{n}$, this will show that (i) $\Rightarrow$ (ii) in Theorem 1. Let $p: X \rightarrow B$ be a Čech cohomology fibration as in the statement of Theorem 2. Note that since we are dealing with local properties it suffices to consider the case in which $B$ is contractible and $p^{-1}(b) \rightarrow X$ induces isomorphisms on Čech cohomology for each $b \in B$. We begin the proof of Theorem 2 by extending $i_{2 k+1}$ to a fiber-preserving embedding $j: X \times B \rightarrow S^{n+2 k+1} \times B$ whose complement is a product.

Lemma 2.1. Let $p: X \rightarrow B$ be a Čech cohomology fibration and let $i: X \rightarrow$ $S^{n} \times B$ be an f.p. fiberwise 1-LCC fiberwise codimension-three embedding, as in the statement of Theorem 2. Let $\gamma: X \rightarrow X \times B$ be given by $\gamma(x)=(x, p(x))$. Then there is a fiberwise 1-LCC fiberwise codimension-three embedding $j: X \times$ $B \rightarrow S^{n+2 k+1} \times B$ such that $\operatorname{proj}_{B} \circ j(x, b)=b$ (i.e., $j$ is fiber-preserving over 
B) and such that

(i) $j \circ \gamma=i_{2 k+1}$, and

(ii) $\operatorname{proj}_{B} \mid:\left(S^{n+2 k+1} \times B-j(X \times B)\right) \rightarrow B$ is a bundle.

Here, $k=\operatorname{dim} B$ as in the statement of Theorem 2 .

Proof. Let $s: B \rightarrow R^{2 k+1}$ be a PL (and therefore 1-LCC) embedding. Writing $i(x)=\left(i^{\prime}(x), p(x)\right) \in S^{n} \times B$, we have a fiber-preserving embedding $j: X \times B \rightarrow$ $S^{n} \times R^{2 k+1} \times B$ given by the formula $j(x, b)=\left(i^{\prime}(x), s \circ p(x)-s(b), b\right)$. The composition $j \circ \gamma$ is clearly the composition of $i$ with (identity) $\times\{0\}: S^{n} \times$ $B \rightarrow S^{n} \times R^{2 k+1} \times B$, i.e., the map sending $(s, b)$ to $(s, 0, b)$. Now, let $H: S^{n} \times R^{2 k+1} \times B \rightarrow S^{n} \times R^{2 k+1} \times B$ be given by the formula $H(p, q, b)=$ $(p, q+s(b), b)$. Then $H$ is a fiber-preserving homeomorphism over $B$ with inverse $H^{-1}(p, q, b)=(p, q-s(b), b)$. Moreover, the composition $H \circ j$ is given by the formula $H \circ j(x, b)=\left(i^{\prime}(x), s \circ p(x), b\right)$, which is the product of a single embedding $X \rightarrow S^{n} \times R^{2 k+1}$ with the identity on $B$. Radially identifying $S^{n} \times R^{2 k+1}$ with a tubular neighborhood of $S^{n}$ in $S^{n+2 k+1}$ and letting $j: X \times B \rightarrow S^{n+2 k+1} \times B$ be the resulting embedding, property (i) in the statement of the lemma is automatic. $H$ is bounded in the $R^{2 k+1}$-direction and therefore extends by the identity outside of the tubular neighborhood of $S^{n} \times B$ to a fiber-preserving homeomorphism $\bar{H}: S^{n+2 k+1} \times B \rightarrow S^{n+2 k+1} \times B$. This verifies property (ii) in the lemma.

The rest of the proof of Theorem 2 consists of proving the following proposition.

Proposition 2.2. Let $p: X \rightarrow B$ be a proper map and let $Y \subset X$ be a closed subset such that the inclusion $p^{-1}(b) \cap Y \rightarrow p^{-1}(b)$ induces isomorphisms on Cech cohomology for each $b \in B$. If $i: X \rightarrow S^{n} \times B$ is a fiberwise 1-LCC fiberwise codimension-three embedding, then for each $l \geq n+2,\left(\left(S^{n+l} \times B\right)-\right.$ $\left.i_{l}(X)\right) \times R^{2}$ is fiber-preserving homeomorphic to $\left(\left(S^{n+l} \times B\right)-i_{l}(Y)\right) \times R^{2}$.

To prove Theorem 2, apply Propositon 2.2 to the pair $(X \times B, \gamma(X))$ and the embedding $j$ constructed in Lemma 2.1. Since the complement of $j(X \times B)$ is a product, the proposition shows that the complement of $j \circ \gamma(X)=$ $i_{2 k+1}(X)$ becomes a product after stabilization and multiplication by $R^{2}$. To prove Proposition 2.2 we first need a lemma.

Lemma 2.3. Let $(X, Y)$ be a pair of compact, connected, finite-dimensional spaces such that the inclusion-induced homomorphism $\check{H}^{*}(X) \rightarrow \check{H}^{*}(Y)$ is an isomorphism. Then the inclusion $\Sigma Y \subset \Sigma X$ is a shape equivalence.

Proof. This follows directly from the cohomological Whitehead theorem in shape theory. See [MS, p. 155] for details.

Definition. If $p: X \rightarrow B$ is a map, then the fiberwise suspension $\Sigma_{B}(p)$ of $p$ is the projection from the double mapping cylinder of $p$ to $B$. In symbols, let 
$D(p)=X \times[-1,1] \amalg B \times\{-1,1\} / \sim$, where $(x, \varepsilon) \sim(p(x), \varepsilon)$ for $\varepsilon= \pm 1$. $\Sigma_{B}(p): D(p) \rightarrow B$ is induced by $(x, t) \rightarrow p(x),(b, \varepsilon) \rightarrow b$. Note that if $p$ is surjective, then for each $b \in B, \Sigma_{B}(p)^{-1}(b)$ is homeomorphic to $\Sigma\left(p^{-1}(b)\right)$. We will have occasion to write $D(p)$ as $M_{+}(p) \cup_{X} M_{-}(p)$ in the obvious way. We will also use $c_{ \pm}: M_{ \pm}(p) \rightarrow B$ to denote the mapping cylinder projections.

Proposition 2.2 follows from Proposition 2.4, which is a stable fibered version of Chapman's finite-dimensional complement theorem. See [MS, p. 264] for the unfibered version.

Proposition 2.4. Let $p: X \rightarrow B$ be a proper map and let $Y \subset X$ be a closed subset such that the inclusion $p^{-1}(b) \cap Y \rightarrow p^{-1}(b)$ is a shape equivalence for each $b \in B$. If $i: X \rightarrow S^{n} \times B$ is a fiber-preserving fiberwise 1-LCC fiberwise codimension-three embedding, then for $l \geq n+1,\left(\left(S^{n+l} \times B\right)-i_{l}(X)\right) \times R^{1}$ is fiber-preserving homeomorphic to $\left(\left(S^{n+l} \times B\right)-i_{l}(Y)\right) \times R^{1}$.

The idea of the proof is quite simple. To avoid losing the reader in notation and details, we first sketch the unfibered version. For this, let $(X, Y)$ be a finite-dimensional pair with $Y \subset X$ a shape equivalence and let $i: X \rightarrow S^{n}$ be a 1-LCC codimension-three embedding. Consider the embedding of $Z=C X \cup_{Y}$ $C Y$ in $S^{n+1}$ obtained by coning $X$ off to the north pole and $Y$ off to the south pole. Let $c_{1}: S^{n+1} \rightarrow S^{n+1} / C X$ and $c_{2}: S^{n+1} \rightarrow S^{n+1} / X \cup_{Y} C Y$ be the quotient maps. Since $C X$ and $X \cup_{Y} C Y$ are codimension-three 1-LCC embedded celllike subsets of $S^{n+1}, c_{1}$ and $c_{2}$ are uniform limits of homeomorphisms. (See Lemma 2.5 below.) $c_{1}$ throws $Z$ onto a copy of $\Sigma Y$ in $S^{n+1}$, while $c_{2}$ throws $Z$ onto a copy of $\Sigma X$ in $S^{n+1}$. This means, in particular, that the complements of $\Sigma X$ and $\Sigma Y$ in $S^{n+1}$ are both homeomorphic to the complement of $Z$ in $S^{n+1}$. If we knew that the resulting embeddings of $\Sigma X$ and $\Sigma Y$ in $S^{n+1}$ were equivalent to the embeddings obtained by coning $X$ and $Y$ off to both poles, then we would be done. An easy way to accomplish this stably is to include the entire picture into a tubular neighborhood of $S^{n+1}$ in $S^{2 n+2}$. The point of doing this is that the decomposition respects the pair $\left(S^{2 n+2}, S^{n+1}\right)$, so $S^{n+1} / C X$ and $S^{n+1} / X \cup_{Y} C Y$ wind up as standard $S^{n+1}$ 's in $S^{2 n+2}$. Klee's trick ([Ru, p. 74] or see below) works as usual to show that any embedding of $\Sigma X$ or $\Sigma Y$ into a standard $S^{n+1}$ in $S^{2 n+2}$ becomes standard in $S^{2 n+2}$. Thus, $\Sigma X$ and $\Sigma Y$ have homeomorphic complements in $S^{2 n+2}$ and $\left(S^{2 n+1}-X\right) \times R^{1}$ is homeomorphic to $\left(S^{2 n+1}-Y\right) \times R^{1}$.

Lemma 2.5. If $X \subset S^{n}, n \geq 5$, is a codimension-three 1-LCC embedded celllike set, then $S^{n} \rightarrow S^{n} / X$ is a uniform limit of homeomorphisms. If $S^{n} \subset S^{n+k}$ is the standard inclusion, then $\left(S^{n+k}, S^{n}\right) \rightarrow\left(S^{n+k} / X, S^{n} / X\right)$ is a uniform limit of homeomorphisms of pairs.

Proof. By [St], 1-LCC embedded compacta satisfy general position with respect to polyhedra. Applying McMillan's cellularity criterion [Ru, p. 178] one easily 


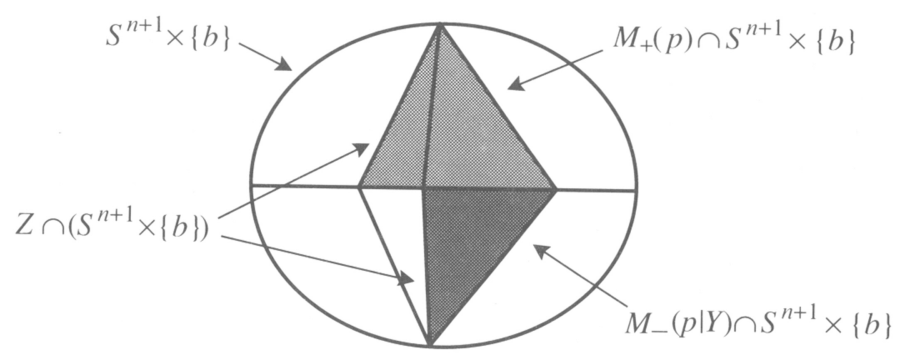

Figure 3

deduces that $X$ is an intersection of nested $n$-cells in $S^{n}$. The result then follows using the usual squeezing argument. See [Ru, p. 44] for details. In the case of pairs, one simply takes care that the squeezing respects the pairs.

Let $X \supset Y$ and $p: X \rightarrow B$ be as in the statement of Proposition 2.4. If $i: X \rightarrow S^{n} \times B$ is a fiberwise 1-LCC fiberwise codimension-three embedding and proj: $S^{n} \times B \rightarrow B$ is the projection, let $\Sigma_{B}$ (proj): $D$ (proj) $\rightarrow B$ be the fiberwise suspension. $D$ (proj) is fiber-preserving homeomorphic to $S^{n+1} \times B$ and contains $D(p), D(p \mid Y)$, and $Z=M_{+}(p) \cup M_{-}(p \mid Y)$ as fiber-preserving subsets. Here, the " \pm " refers to coning from the north and south poles, respectively. The picture is the usual one associated with the Puppe sequence. Figure 3 is a representative slice.

Since the inclusion $p^{-1}(b) \cap Y \rightarrow p^{-1}(b)$ is a shape equivalence, the space $p^{-1}(b) \cup C\left(p^{-1}(b) \cap Y\right)$ is cell-like. Crushing out $M_{+}(p)$ in each fiber turns $Z$ into a copy of $D(p \mid Y)$, while crushing out $Z \cap M_{-}(p)$ turns $Z$ into a copy of $D(p)$. Of course the first fiberwise crushing operation is just

$$
S^{n+1} \times B \rightarrow\left(S^{n+1} \times B\right) \cup_{c_{+}} B
$$

and the second is given by $S^{n+1} \times B \rightarrow\left(S^{n+1} \times B\right) \cup_{\left(c_{-} \mid\left(Z \cap M_{-}(p)\right)\right)} B$. By Lemma 2.5 , crushing $M_{+}(p) \cap\left(S^{n+1} \times\{b\}\right)$ or $\left(Z \cap M_{-}(p)\right) \cap\left(S^{n+1} \times\{b\}\right)$ out of $S^{n+1} \times\{b\}$ results in a space homeomorphic to $S^{n+1}$. We now prove that this identification can be made fiber-preserving.

Theorem 2.6 [EK, Remark on p. 87]. If $(M, N)$ is a locally flat manifold pair, the group $\mathscr{H}(M, N)$ of self-homeomorphisms of $(M, N)$ is locally contractible.

It is easy to extend Theorem 1.1 to a theorem for pairs. Thus, we say that $f:\left(X, X_{0}\right) \rightarrow Y$ is a completely regular pair of maps if for each point $y \in Y$ and $\varepsilon>0$ there is $\delta>0$ such that if $y^{\prime} \in Y$ and $d\left(y^{\prime}, y\right)<\delta$, then there is a homeomorphism $h:\left(f^{-1}\left(y^{\prime}\right), f^{-1}\left(y^{\prime}\right) \cap X_{0}\right) \rightarrow\left(f^{-1}(y), f^{-1}(y) \cap X_{0}\right)$ with $d(h, \mathrm{id})<\varepsilon$.

The proof in $[\mathrm{DH}]$ then allows us to conclude that if $Y$ is finite dimensional and $f:\left(X, X_{0}\right) \rightarrow Y$ is a completely regular pair of maps with $f^{-1}(y)$ a closed manifold and $f^{-1}(y) \cap X_{0}$ a locally flat submanifold for each $y \in Y$, then for 
each $y \in Y$ there is a neighborhood $U$ of $y$ in $Y$ such that $\left(f^{-1}(U), f^{-1}(U)\right.$ ก $\left.X_{0}\right)$ is fiber-preserving homeomorphic to $\left(f^{-1}(y), f^{-1}(y) \cap X_{0}\right) \times U$.

Proposition 2.7. Let $M$ and $N$ be closed $n$-manifolds and let $B$ be a finite complex. If $f: M \times B \rightarrow N \times B$ is a fiber-preserving map such that $f \mid M \times\{b\}$ is a uniform limit of homeomorphisms for each $b \in B$, then $f$ is a uniform limit of fiber-preserving homeomorphisms.

Proof. We proceed by induction on $\operatorname{dim} B$. Assume that the proposition is true for $\operatorname{dim} B<k$ and let $B=B^{k}$ be a finite polyhedron with $f: M \times B \rightarrow N \times B$ a fiber-preserving map as in the statement of the proposition. Let $\varepsilon>0$ be given and choose $\delta>0$ so that every map $\alpha: S^{k-1} \rightarrow \mathscr{H}(N)$ with $d(\alpha(x)$, id $)<\delta$ for all $x \in S^{k-1}$ extends to $\bar{\alpha}: D^{k} \rightarrow \mathscr{H}(N)$ with $d(\bar{\alpha}$, id $)<\varepsilon / 2$. We may assume that $\delta<\varepsilon / 2$.

Triangulate $B$ by simplexes so small that $d\left(f_{b}, f_{b^{\prime}}\right)<\delta / 3$ whenever $b, b^{\prime} \in$ $\Delta^{1}, \Delta^{1}$ a simplex of $B$. Here we write $f_{b}$ for $f \mid M \times\{b\}$ considered as a map from $M$ to $N$. By induction, there is a fiber-preserving homeomorphism $h: M \times B^{(k-1)} \rightarrow N \times B^{(k-1)}$ with $d\left(h, f \mid M \times B^{(k-1)}\right)<\delta / 3$. Let $\Delta^{k}$ be a $k$-simplex in $B$. Choose $b_{0} \in \Delta^{k}$ and let $h_{b_{0}}: M \rightarrow N$ be a homeomorphism which $\delta / 3$-approximates $f_{b}$. If $b^{\prime} \in \partial \Delta^{k}$, we have

$$
d\left(h_{b_{0}}, h_{b^{\prime}}\right) \leq d\left(h_{b_{0}}, f_{b}\right)+d\left(f_{b}, f_{b^{\prime}}\right)+d\left(f_{b^{\prime}}, h_{b^{\prime}}\right) \leq \delta / 3+\delta / 3+\delta / 3=\delta .
$$

Define $\alpha: \partial \Delta^{k} \rightarrow \mathscr{H}(N)$ by $\alpha\left(b^{\prime}\right)=h_{b^{\prime}} \circ h_{b_{0}}^{-1}, d\left(h_{b^{\prime}} \circ h_{b_{0}}^{-1}\right.$, id $)=d\left(h_{b^{\prime}}, h_{b_{0}}\right)<$ $\delta$, so $\alpha$ has an extension $\bar{\alpha}: \Delta^{k} \rightarrow \mathscr{H}(N), h_{b}=\bar{\alpha}(b) \circ h_{b_{0}}$ gives an extension of $h$ over $\Delta^{k}$. Now, for $b \in B$ we have

$$
\begin{aligned}
d\left(h_{b}, f_{b}\right) & =d\left(\bar{\alpha}(b) \circ h_{b_{0}}, f_{b}\right)=d\left(\bar{\alpha}(b), f_{b} \circ h_{b_{0}}^{-1}\right) \\
& \leq d(\bar{\alpha}(b), \mathrm{id})+d\left(\mathrm{id}, f_{b} \circ h_{b_{0}}^{-1}\right) \leq \varepsilon / 2+d\left(\mathrm{id}, f_{b} \circ h_{b_{0}}^{-1}\right) \\
& =\varepsilon / 2+d\left(h_{b_{0}}, f_{b}\right) \leq \varepsilon / 2+d\left(h_{b_{0}}, f_{b_{0}}\right)+d\left(f_{b_{0}}, f_{b}\right) \\
& \leq \varepsilon / 2+2 \delta / 3<\varepsilon .
\end{aligned}
$$

Repeating this for each $k$-simplex of $B$ completes the inductive step and the proof.

Remark. Proposition 2.7 is also true for pairs. Thus, if $\left(M, M_{0}\right)$ and $\left(N, N_{0}\right)$ are locally flat manifold pairs, $B$ is a finite complex, and $f:\left(M, M_{0}\right) \times B \rightarrow$ $\left(N, N_{0}\right) \times B$ is a fiber-preserving map such that $f_{b}:\left(M, M_{0}\right) \rightarrow\left(N, N_{0}\right)$ is a uniform limit of homeomorphisms of pairs for each $b \in B$, then $f$ is a uniform limit of fiber-preserving homeomorphisms of pairs.

Proposition 2.8. Let $X$ and $B$ be compact with $p: X \rightarrow B$ a map. Let $i, j: X \rightarrow R^{n} \times B \subset S^{n} \times B$ be fiber-preserving embeddings. Then there is a fiber-preserving homeomorphism $H: R^{n} \times R^{n} \times B \rightarrow R^{n} \times R^{n} \times B$ with compact support such that $H(i(x), 0, p(x))=(0, j(x), p(x))$. 
Proof. The proof is the same as the usual version of Klee's trick. Consider fiberpreserving embeddings $\alpha=\left(\operatorname{proj}_{R^{n}} \times 0 \times \operatorname{proj}_{B}\right) \circ j: X \rightarrow R^{n} \times 0 \times B$ and $\beta=$ $\left(0 \times \operatorname{proj}_{R^{n}} \times \operatorname{proj}_{B}\right) \circ i: X \rightarrow 0 \times R^{n} \times B$. Let $u=\beta \circ \alpha^{-1}: \alpha(X) \rightarrow \beta(X)$. Note that $u$ has domain a subset of $R^{n} \times 0 \times B$ and range a subset of $0 \times R^{n} \times B$ and that $u$ has the form $u(w, 0, b)=\left(0, u_{1}(w, b), b\right)$. Let $\bar{u}_{1}$ be an extension of $u_{1}$ to all of $R^{n} \times B$. We may arrange for $u_{1}$ to have compact support. Now define $U^{\prime}: R^{n} \times R^{n} \times B \rightarrow R^{n} \times R^{n} \times B$ by the formula $U^{\prime}(w, z, b)=(w, z+$ $\left.u_{1}(w, b), b\right)$. $U^{\prime}$ pushes $\alpha(X)$ to the set $\left\{\left(w, u_{1}(w, b), b\right) \mid(w, b) \in R^{n} \times B\right)$. This set is a fiber-preserving graph of $\bar{u}_{1}$ : For each fixed $b$, it is the graph of $\bar{u}_{1} \mid R^{n} \times\{b\} . U^{\prime}$ is a bounded homeomorphism, so the usual radial squeezing trick (see [C, Lemma 5]) done fiberwise yields a homeomorphism $U$ which has compact support and which equals $U^{\prime}$ in a neighborhood of $\alpha(X)$. Similarly, one constructs a fiber-preserving homeomorphism $V$ with bounded support pushing $\beta(X)$ onto an extension of the graph of $\alpha \circ \beta^{-1} . H=V^{-1} \circ U$ is then the desired homeomorphism with compact support throwing $\alpha(X)$ onto $\beta(X)$. The details are as in [Ru, p. 74].

The proof of Proposition 2.4 now follows exactly as advertised. Let $X \supset Y$ and $p: X \rightarrow B$ be as in the statement of Proposition 2.4. If $i: X \rightarrow R^{n} \times$ $B \subset S^{n} \times B$ is a fiberwise 1-LCC fiberwise codimension-three embedding, then consider $i_{n+1}: X \rightarrow S^{2 n+1} \times B$. Then $D(p), D(p \mid Y)$, and $Z$ are all contained in $S^{n+1} \times B \subset S^{2 n+2} \times B$. Crushing, as in the discussion following Lemma 2.5, and applying the Remark following Proposition 2.7, we see that $\left(S^{2 n+2} \times B\right)-$ $D(p)$ and $\left(S^{2 n+2} \times B\right)-D(p \mid Y)$ are both homeomorphic to $\left(S^{2 n+2} \times B\right)-Z$. Proposition 2.8 shows that any two embeddings of $Z$ into $\left(S^{n+1} \times B\right) \subset\left(S^{2 n+2} \times\right.$ $B)$ are equivalent, so $\left(S^{2 n+2} \times B\right)-D(p)$ and $\left(S^{2 n+2} \times B\right)-D(p \mid Y)$ are fiberpreserving homeomorphic. This shows that $\left(\left(S^{2 n+1} \times B\right)-i_{n+1}(X)\right) \times R^{1}$ is fiber-preserving homeomorphic to $\left(\left(S^{2 n+1} \times B\right)-i_{n+1}(Y)\right) \times R^{1}$.

Although Proposition 2.4 should be regarded as a fibered complement theorem, we note that it starts with weak data in that we only require that the inclusion be a shape equivalence on each fiber. It follows from the complement theorem of $[\mathrm{CM}]$ that after fiberwise suspension such an inclusion becomes a fiber shape equivalence. One wonders if the suspension is necessary.

Corollary 2.9. Let $X$ and $Y$ be compact finite-dimensional metric spaces and let $p: X \rightarrow B$ and $q: Y \rightarrow B$ be maps, $B$ a finite polyhedron. If $f: X \rightarrow Y$ is a map with $q \circ f=p$ such that $f \mid: p^{-1}(b) \rightarrow q^{-1}(b)$ induces isomorphisms on Cech cohomology for each $b \in B$, then the fiberwise suspension $\Sigma_{B}(f): D(X) \rightarrow$ $D(Y)$ is a fiber shape equivalence in the sense of [CM].

Another consequence of the argument is the following generalization of the classical Vietoris-Begle theorem.

Proposition 2.10. Let $X, Y$, and $B$ be compact metric spaces and let $p: X \rightarrow B$ and $q: Y \rightarrow B$ be maps. If $f: X \rightarrow Y$ is a map with $q \circ f=p$ such that 
$f \mid: p^{-1}(b) \rightarrow q^{-1}(b)$ is a Čech cohomology equivalence for each $b \in B$ then $f$ is a Čech cohomology equivalence.

Proof. Using mapping cylinders, we may assume that $f$ is an inclusion. The construction used in proving Proposition 2.4 yields a compact space $Z$, a map $r: Z \rightarrow B$, and fiber-preserving acyclic maps $c_{1}: Z \rightarrow D(X), c_{2}: Z \rightarrow D(Y)$. By the classical Vietoris-Begle theorem, the maps $c_{1}$ are Čech cohomology equivalences. Since $\Sigma_{B}(f) \circ c_{1}$ is homotopic to $c_{2}, \Sigma_{B}(f): D(X) \rightarrow D(Y)$ is also a Čech cohomology equivalence. We have commuting diagrams of cofibrations, where $\Sigma$ denotes the usual (nonfiberwise) suspension and $B_{+}$and $B_{-}$ are the two copies of $B$ in $D(X)$ and $D(Y)$ :

$$
\begin{array}{ccccc}
B_{+} & \rightarrow & D(X) & \rightarrow & D(X) / B_{+} \\
\downarrow \text { id } & & \downarrow \Sigma_{B}(f) & & \downarrow \\
B_{+} & \rightarrow & D(Y) & \rightarrow & D(Y) / B_{+}
\end{array}
$$

and

$$
\begin{array}{llcl}
B_{-} \rightarrow & \left(D(X) / B_{+}\right) / B_{-} \rightarrow & \Sigma(X) \\
\downarrow \text { id } & \downarrow & & \downarrow \Sigma(f) \\
B_{-} \rightarrow & \left(D(Y) / B_{+}\right) / B_{-} \rightarrow & \Sigma(Y)
\end{array}
$$

Passing to Čech cohomology and applying the Five Lemma shows that $\Sigma(f)$, and therefore $f$, is a Čech cohomology equivalence.

\section{A DUALITY THEOREM}

Our proof that $p: X \rightarrow B$ is a Čech cohomology fibration whenever $\left(S^{n} \times\right.$ $B)-X \rightarrow B$ is a Hurewicz fibration requires the duality theorem stated as Theorem 3. We give a proof.

Proof (Theorem 3). Write $X=\bigcap N_{i}$, where $N_{1} \supset N_{2} \supset N_{3} \supset \cdots$ are codimension-0 PL submanifolds of $M$ meeting $\partial M, P, Q$, and $\partial P=\partial Q$ regularly. We will write $\partial X$ for $X \cap \partial M$. The following is a picture (see Figure 4). The picture is slightly misleading in that it is perfectly permissible for $X$ to meet $\partial P=\partial Q$. We write

$$
\begin{aligned}
& \check{H}^{k}(M, Q \cup X) \cong \lim _{\longrightarrow} H^{k}\left(M, Q \cup N_{i}\right) \\
& \quad \cong \lim _{\longrightarrow} H^{k}\left(M-\operatorname{int} N_{i},\left(Q-\operatorname{int}_{\partial M}\left(\partial N_{i} \cap Q\right)\right) \cup\left(\partial N_{i}-\left(\partial N_{i} \cap \partial M\right)\right)\right) \\
& \quad \cong \lim _{n-k}\left(M-\operatorname{int}_{M} N_{i},\left(P-\operatorname{int}_{\partial M}\left(\partial N_{j} \cap P\right)\right)\right) \\
& \quad \cong H_{n-k}(M-X, P-(X \cap P)) . \quad \square
\end{aligned}
$$

We now prove that (ii) $\Rightarrow$ (i) in Theorem 1 . With notation as in the statement of Theorem 1, we must show that for each $b \in B$ there is a contractible neighborhood $U$ of $b$ such that $p^{-1}\left(b^{\prime}\right) \rightarrow p^{-1}(U)$ is a Čech cohomology equivalence for each $b^{\prime} \in U$. 


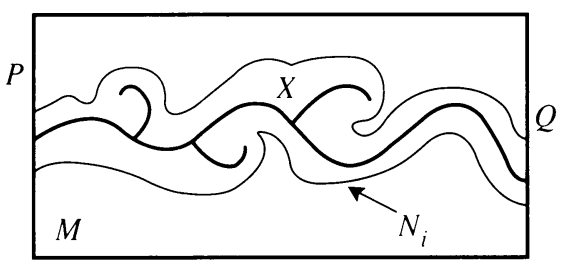

FIGURE 4

Case I. Let $B=\Delta^{k}$ and let $b$ be a vertex of $\Delta^{k}$. We will show that $p^{-1}(b) \rightarrow$ $p^{-1}\left(\Delta^{k}\right)$ induces an isomorphism on Čech cohomology.

Proof (Case I). We proceed by induction, assuming that the result is true over proper faces of $\Delta^{k}$. Let $p: X \rightarrow \Delta^{k}$ be the given map and let $i: X \rightarrow S^{n} \times \Delta^{k}$ be a fiber-preserving map such that $S^{n} \times \Delta^{k}-i(X) \rightarrow \Delta^{k}$ is a Hurewicz fibration. Let $Q^{\prime}$ be a $(k-1)$-dimensional face of $\Delta^{k}$ and let $P^{\prime}$ be the closure of $\Delta^{k}-Q^{\prime}$. Let $Q=S^{n} \times Q^{\prime}$ and let $P=S^{n} \times P^{\prime}$. Then $\partial\left(S^{n} \times \Delta^{k}\right)=P \cup Q$. Applying Theorem 3, we have $\check{H}^{k}\left(S^{n} \times \Delta^{k}, Q \cup X\right) \cong H_{n-k}\left(S^{n} \times \Delta^{k}-X, P-(P \cap \partial X)\right)$. Since $S^{n} \times \Delta^{k}-i(X) \rightarrow \Delta^{k}$ is a Hurewicz fibration, the group on the right is zero for all $k$ and we have $\breve{H}^{k}\left(S^{n} \times \Delta^{k}, Q \cup X\right)=0$ for all $k$. This implies that $\check{H}^{k}(Q \cup X) \cong \check{H}^{k}\left(S^{n}\right)$ for all $k$. Since $\check{H}^{k}(Q) \cong \check{H}^{k}\left(S^{n}\right)$, we see that $\breve{H}^{k}(Q \cup X, Q) \cong \check{H}^{k}(X, Q \cap X)=0$ for all $k$. Thus, $\check{H}^{k}(Q) \rightarrow \breve{H}^{k}(Q \cap X)$ is an isomorphism for all $k$. By our inductive assumption, $\check{H}^{k}(Q \cap X) \rightarrow \breve{H}^{k}\left(p^{-1}(b)\right)$ is an isomorphism for all $k$, so Case I is proved.

Case II. Let $B=\Delta^{k}$ and let $b$ be an interior point of $\Delta^{k}$.

Proof (Case II). Starting from $b$ produces a triangulation of $\Delta^{k}$ in which $b$ is a vertex. Case II is now a simple application of the Mayer-Vietoris theorem using Case I.

Case III. Let $B=\Delta^{k}$ and let $b$ be a point of $\partial \Delta^{k}$.

Proof (Case III). In proving Case I, we showed that if $\Delta^{k-1}$ is a face of $\Delta^{k}$, then $p^{-1}\left(\Delta^{k-1}\right) \rightarrow p^{-1}\left(\Delta^{k}\right)$ induces isomorphisms on Čech cohomology. If $b$ is an interior point of $\Delta^{l} \subset \Delta^{k}, p^{-1}(b) \rightarrow p^{-1}\left(\Delta^{l}\right)$ and $p^{-1}\left(\Delta^{l}\right) \rightarrow p^{-1}\left(\Delta^{k}\right)$ both induce isomorphisms on Čech cohomology.

Case IV. The general case.

Proof. If $B$ is a finite polyhedron and $b \in B$, then $b$ has a closed neighborhood which is a simplicial cone with vertex $b$. That $p^{-1}(b) \rightarrow p^{-1}(B)$ induces isomorphisms on Cech cohomology follows from Case I and the Mayer-Vietoris theorem. If $b^{\prime}$ is in the cone neighborhood, then there is a simplex $\sigma$ of $B$ containing both $b$ and $b^{\prime}$. Since $p^{-1}(b) \rightarrow p^{-1}(B)$ and $p^{-1}(b) \rightarrow p^{-1}(\sigma)$ induce isomorphisms on Čech cohomology, so does $p^{-1}(\sigma) \rightarrow p^{-1}(B)$. Since $p^{-1}\left(b^{\prime}\right) \rightarrow p^{-1}(\sigma)$ induces isomorphisms on Čech cohomology by Case III, so does $p^{-1}\left(b^{\prime}\right) \rightarrow p^{-1}(B)$. 


\section{EXAMPLES, CONJECTURES, GENERALIZATIONS, AND THE PROOF OF THEOREM 4}

Theorem 1 is a stable result. We conjecture that it can be improved enormously:

Conjecture. If $p: X \rightarrow B$ is a Čech cohomology fibration from a finite-dimensional metric space to a finite complex and $i: X \rightarrow S^{n} \times B$ is a fiber-preserving embedding which is codimension-three and 1-LCC on each fiber, then

$$
\operatorname{proj}_{B} \mid:\left(S^{n} \times B_{-i}(X)\right) \rightarrow B
$$

is a Hurewicz fibration.

Remark. Some tameness condition is necessary. If $\alpha:[0,1] \rightarrow S^{3}$ is the Fox-Artin arc (see [Ru, p. 68]), there is a continuous family of embeddings $\alpha_{t}:[0,1] \rightarrow S^{3}$ such that $\alpha_{t}$ is tame for $t<1$ and $\alpha_{1}=\alpha .\left(S^{3} \times I-\alpha(I \times I)\right) \rightarrow$ $I$ is not a Hurewicz fibration because the fibers have different homotopy types. Of course, including $S^{3}$ into a larger sphere as in the proof of our main theorem removes this difficulty.

As stated, Theorems 1 and 2 apply only to maps from finite-dimensional spaces to finite polyhedra. It is customary to state theorems of this sort at least for bases which are Euclidean neighborhood retracts (ENR's) as in [D]. Everything we have discussed generalizes easily to this case except for the definition of Čech cohomology fibration itself. The problem is that the definition given requires that the base space have arbitrarily small contractible covers. This is sufficient to guarantee that the base space be an ENR, but it is by no means necessary. A related problem is that it is not obvious from the original definition that Čech cohomology fibrations are preserved under pullbacks. A version of this is a consequence of Theorem 1:

Proposition 4.1. Let $p: E \rightarrow B$ be a Čech cohomology fibration. If $K$ is a finite polyhedron and $f: K \rightarrow B$ is a map, then the induced map $p^{*}: f^{*} E \rightarrow K$ from the pullback to $K$ is a Čech cohomology fibration.

Proof. By our main results, there is a fiber-preserving embedding $i: E \rightarrow S^{n} \times B$ such that $\left(\left(S^{n} \times B\right)-i(E)\right) \times R^{2} \rightarrow B$ is a locally trivial fiber bundle. Pulling back the pair $\left(S^{n} \times B, i(E)\right)$ over $f$ gives an embedding $i^{*}: f^{*} E \rightarrow S^{n} \times K$ for which the complement has the same property-implying that $f^{*} E \rightarrow K$ is a Čech cohomology fibration.

Remark. We could now define a Čech cohomology fibration to be a map $p$ from a compact metric space $X$ to a compact ENR $Y$ such that for every map $\alpha: \Delta^{k} \rightarrow Y$ the pullback $p^{*}: \alpha^{*} X \rightarrow \Delta^{k}$ is a Cech cohomology fibration in the original sense. Standard properties of pullbacks show that Theorems 1 and 2 extend immediately to this extra generality.

We begin our proof of Theorem 4 with the following proposition.

Proposition 4.2. Let $p: E \rightarrow[0,1]$ be an $n$-manifold fibration, $n \neq 3$. If $t_{0} \in[0,1]$ and $D$ is a compact subset of $\operatorname{int}\left(p^{-1}\left(t_{0}\right)\right)$, then there exist an open 
neighborhood $U$ of $D$ in $p^{-1}\left(t_{0}\right), a \delta>0$ and a continuous family of open embeddings $h_{t}: U \rightarrow p^{-1}(t), t \in\left(t_{0}-\delta, t_{0}+\delta\right) \cap[0,1]$, with $h_{t_{0}}=\mathrm{id}_{U}$.

Proof. The main theorem of $[\mathrm{ChF}]$ shows that small homotopy equivalences between $n$-manifolds, $n \geq 5$, can be approximated by homeomorphisms. The argument is a handle induction and applies to an open neighborhood $U$ of $D$ to give the stated result separately on fibers. One then uses the local contractibility of the homeomorphism group [EK] to obtain a continuous family of embeddings. In dimensions 4 and 2, results of Quinn $\left[\mathrm{Q}_{3}\right]$ and Jakobsche [J] replace $[\mathrm{ChF}]$.

Proof of Theorem 4(i). If $p^{-1}\left(t_{0}\right)$ is a compact $n$-manifold with boundary, let $C$ be a collar on $\partial p^{-1}\left(t_{0}\right)$ and let $D$ be the closure of $p^{-1}\left(t_{0}\right)-C$. If $C$ is chosen so that $\partial C$ is collared in $D$, then $D$ is homeomorphic to $p^{-1}\left(t_{0}\right)$. By Proposition 4.2, there is a $\delta>0$ so that $D$ embeds in $p^{-1}(t)$ for every $t \in\left(t_{0}-\delta, t_{0}+\delta\right) \cap[0,1]$. Since this embedding is a homotopy equivalence for each $t \in\left(t_{0}-\delta, t_{0}+\delta\right) \cap[0,1], H_{*}\left(p^{-1}(t)-\operatorname{int}\left(h_{t}(D)\right), h_{t}(\partial D)\right)=0$. By duality, $H_{*}\left(p^{-1}(t)-\operatorname{int}\left(h_{t}(D)\right), \partial p^{-1}(t)\right)=0$ for each $t \in\left(t_{0}-\delta, t_{0}+\delta\right) \cap[0,1]$, so

$$
\begin{aligned}
H_{*}\left(\partial p^{-1}\left(t_{0}\right)\right) & \cong H_{*}\left(h_{t}(\partial D)\right) \cong H_{*}\left(p^{-1}(t)-\operatorname{int}\left(h_{t}(D)\right)\right) \\
& \cong H_{*}\left(\partial p^{-1}(t)\right) \text { for all } t \in\left(t_{0}-\delta, t_{0}+\delta\right) \cap[0,1] .
\end{aligned}
$$

Corollary 4.3. If $M$ and $N$ deform to each other through open n-manifolds, then $M$ is orientable if and only if $N$ is.

Proof. By crossing with a high-dimensional sphere, we may assume that the fiber dimension is $\geq 6$. Choose a section and consider this to define a basepoint in each fiber. If $[\alpha] \in \pi_{1}\left(p^{-1}(t)\right)$, let $\left[\alpha_{t^{\prime}}\right] \in \pi_{1}\left(p^{-1}\left(t^{\prime}\right)\right)$ be the corresponding element. Proposition 4.2 applies to a neighborhood of a representative of $[\alpha]$ to show that $[\alpha]$ preserves orientation if and only if $\left[\alpha_{t^{\prime}}\right]$ does for each $t^{\prime}$ in a neighborhood of $t$ in $[0,1]$. Applied separately to each element of $\pi_{1}\left(p^{-1}(t)\right)$, this proves the corollary.

Notation. For the remainder of this section, $H_{k}(M)$ and $H^{k}(M)$ will mean homology and cohomology with coefficients in a field $\mathbb{F}$ over which the manifold $M$ is orientable.

Our proof of Theorem 4 will rely on the following propositions.

Proposition 4.4. If $M^{n}$ is a connected orientable open manifold, $n \geq 6$, with $\operatorname{dim}_{\mathbb{F}} H_{n-1}(M)<\infty$, then there exists a compact submanifold $N^{n} \subset M$ such that $H_{n-1}(N) \rightarrow H_{n-1}(M)$ is an isomorphism.

Proof. By [KS], $M$ has a handle decomposition. Choose a compact submanifold $N^{\prime}$ of $M$ large enough that $H_{n-1}\left(N^{\prime}\right) \rightarrow H_{n-1}(M)$ is an epimorphism. By Alexander Duality, $H_{0}^{l f}\left(M-\stackrel{\circ}{N^{\prime}}\right) \cong H^{n}\left(M, N^{\prime}\right), \operatorname{Dim}_{\mathbb{F}} H_{0}^{l f}\left(M-\stackrel{\circ}{N}^{\prime}\right)$ measures 
the number of compact components of $M-\stackrel{\circ}{N}^{\prime}$ and the exact sequence of the pair $\left(M, N^{\prime}\right)$ shows that this number is finite. Let

$$
N=N^{\prime} \cup\left\{\text { compact components of } M-N^{\prime}\right\} \text {. }
$$

We now have $H_{0}^{l f}(M-\stackrel{\circ}{N})=H^{n}(M, N)=H_{n}(M, N)=0$. Since $N \supset N^{\prime}$, $H_{n-1}(N) \rightarrow H_{n-1}(M)$ is still an epimorphism. The exact sequence

$$
\cdots \rightarrow H_{n}(M, N) \rightarrow H_{n-1}(N) \rightarrow H_{n-1}(M) \rightarrow \cdots
$$

shows that $H_{n-1}(N) \rightarrow H_{n-1}(M)$ is an isomorphism.

Addendum. The proof above shows that $H_{n-1}(N) \rightarrow H_{n-1}(M)$ is an isomorphism whenever:

(i) $H_{n-1}(N) \rightarrow H_{n-1}(M)$ is surjective and

(ii) $M-\stackrel{\circ}{N}$ has no compact components.

This means that for $n>2$ we can always find $N$ so that $H_{n-1}(N) \rightarrow H_{n-1}(M)$ is an isomorphism and $H_{0}(\partial N) \rightarrow H_{0}(M-\stackrel{\circ}{N})$ is an isomorphism.

Proof. Since $M$ is connected, every component of $M-\stackrel{\circ}{N}$ meets $\partial N$. If a single component of $M-\stackrel{\circ}{N}$ contains more than one component of $\partial N$, run arcs between these components in $M-\stackrel{\circ}{N}$ and add regular neighborhoods of these arcs to $N$. We now have $H_{0}(\partial N) \rightarrow H_{0}(M-\stackrel{\circ}{N})$ an isomorphism. Since (i) and (ii) still hold, $H_{n-1}(N) \rightarrow H_{n-1}(M)$ remains an isomorphism.

Proposition 4.5. Let $M$ be a connected orientable open n-manifold and let $N \subset M$ be a compact $n$-manifold with $H_{n-1}(N) \rightarrow H_{n-1}(M)$ and $H_{0}(\partial N) \rightarrow$ $H_{0}(M-\stackrel{\circ}{N})$ isomorphisms. Then the number of ends of $M$ is equal to the number of boundary components of $N$.

Proof. Since $H_{0}^{l f}(M-\stackrel{\circ}{N})=H^{n}(M, N)=0$, the components of $M-\stackrel{\circ}{N}$ are noncompact. We have a commuting diagram:

$$
\begin{aligned}
& 0
\end{aligned}
$$

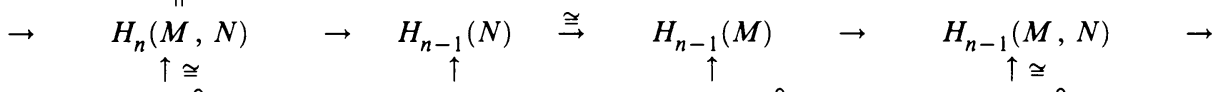

$$
\begin{aligned}
& \rightarrow H_{n}(M-\stackrel{\circ}{N}, \partial N) \rightarrow H_{n-1}(\partial N) \rightarrow H_{n-1}(M-\stackrel{\circ}{N}) \rightarrow H_{n-1}(M-\stackrel{\circ}{N}, \partial N) \rightarrow
\end{aligned}
$$

which shows that $H_{n-1}(\partial N) \rightarrow H_{n-1}(M-\stackrel{\circ}{N})$ is an isomorphism. Since this must hold componentwise, the result follows from:

Proposition 4.6. If $W$ is a connected noncompact n-manifold with compact connected nonempty boundary such that $H_{n-1}(\partial W) \rightarrow H_{n-1}(W)$ is an isomorphism, then $W$ is 1-ended.

Proof. Suppose that $W$ has more than one end. Then there is a neighborhood of infinity in $W$ with more than one noncompact component. We may therefore choose a disconnected manifold neighborhood $V$ of infinity which has 


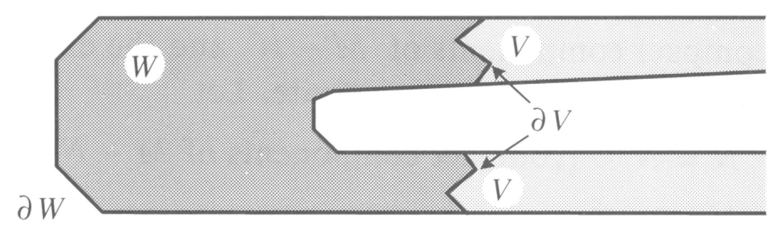

FIGURE 5

compact boundary, no compact components, and a single connected complementary domain. Since $W$ is connected, such a $V$ has disconnected boundary. The exact sequence

$$
0=H_{n}(W, \partial V) \rightarrow H_{n-1}(\partial V) \rightarrow H_{n-1}(W) \rightarrow \cdots
$$

shows that $\operatorname{dim}_{\mathbb{F}} H_{n-1}(W)$ is at least two, contradicting the assumption that $\mathbb{F}=H_{n-1}(\partial W)$ is isomorphic to $H_{n-1}(W)$. See Figure 5 .

Proof (Theorem 4(ii)). By crossing with a high-dimensional sphere, we may assume that the fiber dimension is at least six. Given $t_{0} \in[0,1]$, choose a compact submanifold $V$ of $p^{-1}\left(t_{0}\right)$ such that $H_{n-1}(V) \rightarrow H_{n-1}\left(p^{-1}\left(t_{0}\right)\right)$ is an isomorphism. By Proposition 4.2 there is a $\delta>0$ such that $V$ embeds in $p^{-1}(t)$ for $t \in\left(t_{0}-\delta, t_{0}+\delta\right) \cap[0,1]$. By Proposition 4.5, the number of ends of $p^{-1}(t)$ is equal to the number of boundary components of $V$ for each $t \in\left(t_{0}-\delta, t_{0}+\delta\right) \cap[0,1]$. Theorem 4 follows by compactness.

We close with some questions.

Question 1. Is Theorem 4 true without the hypothesis on $H_{n-1}(M)$ ?

Question 2. If $p: E \rightarrow B$ is a Hurewicz fibration with open manifold fibers, then if $p \circ \operatorname{proj}_{E}: E \times R^{k} \rightarrow B$ a locally trivial bundle for some $k$ ?

\section{REFERENCES}

[CM] M. Clapp and L. Montejano, Parameterized shape theory, Glas. Mat. Ser. III 40 (1985), 215-241.

[Ch $\mathrm{Ch}_{1}$ T. A. Chapman, Proper fibrations with n-manifold fibers, Indiana Univ. Math. J. 30 (1981), 79-102.

$\left[\mathrm{Ch}_{2}\right]$ _ Piecewise linear fibrations, Pacific J. Math. 128 (1987), 223-250.

[ChF] T. A. Chapman and S. C. Ferry, Approximating homotopy equivalences by homeomorphisms, Amer. J. Math. 101 (1979), 583-607.

[C] E. H. Connell, Approximating stable homeomorphisms by piecewise linear ones, Ann. of Math. (2) 78 (1963), 326-338.

[D] A. Dold, The fixed-point index of fiber-preserving maps, Invent. Math. 25 (1974), 281-297.

[DH] E. Dyer and M.-E. Hamstrom, Completely regular mappings, Fund. Math. 45 (1958), 104118.

[Du] J. Dugundji, Topology, Allyn and Bacon, Boston, Mass., 1965.

[EK] R. D. Edwards and R. C. Kirby, Deformations of spaces of embeddings, Ann. of Math. (2) 93 (1971), 63-88. 
[H] A. Hatcher, Higher simple homotopy theory, Ann. of Math. (2) 102 (1975), 101-137.

[J] W. Jakobsche, Approximating homotopy equivalences of surfaces by homeomorphisms, preprint.

[KS] R. Kirby and L. C. Siebenmann, Foundational essays on topological manifolds, smoothings, and triangulations, Princeton Univ. Press, Princeton, N.J., 1977.

[Mc] D. McDuff, Configuration spaces of positive and negative particles, Topology 14 (1975), 91-107.

[McS] D. McDuff and G. Segal, Homology fibrations and the "Group-Completion" Theorem, Invent. Math. 31 (1976), 279-284.

[MS] S. Mardesic and J. Segal., Shape theory, North-Holland, Amsterdam, 1982.

[Q $Q_{1}$ ] F. Quinn, Ends of maps. I, Ann. of Math. (2) 110 (1979), 275-331.

$\left[\mathrm{Q}_{2}\right] \ldots$, An obstruction to the resolution of homology manifolds, Michigan Math. J. 301 (1987), 285-292.

$\left[\mathrm{Q}_{3}\right]$ _ Ends of maps. III, J. Differential Geom. 17 (1982), 503-521.

[R] F. Raymond, Local triviality for Hurewicz fiberings of manifolds, Topology 3 (1965), 43-57.

[Ru] T. B. Rushing, Topological embeddings, Academic Press, New York, 1973.

[S] L. C. Siebenmann, Approximating cellular maps by homeomorphisms, Topology 11 (1972), 271-294.

[Št] M. A. Štanko, Embedding of compacta in euclidean space, Math. USSR-Sb. 12 (1970), 234-255.

[W] F. Waldhausen, Algebraic K-theory of spaces, Lecture Notes in Math., vol. 1126, SpringerVerlag, pp. 318-419.

Department of Mathematics, State University of New York at Binghamton, BingHAMTON, NEW YORK 13901 